\title{
11 The Study of Genuine and Simulated Notes
}

Shneidman and Farberow (1957b) published a sample of suicide notes that they found in the files of the Los Angeles medical examiner's office. They matched the 33 genuine suicide notes with 33 "simulated" suicide notes, that is, suicide notes solicited from people who were not contemplating suicide who were asked to pretend that they were going to complete suicide and to write a suicide note. Since then, many papers have appeared comparing this set of genuine and simulated suicide notes. ${ }^{33}$

Lester (1988a) has argued that this comparison does not shed light on the mind of suicidal individuals but rather examines whether nonsuicidal individuals have any insight into the suicidal mind. Since many studies have found differences between the genuine and the simulated suicide notes, the evidence is that nonsuicidal individuals do not have good insights into the suicidal mind. For example, a small proportion (15\%) of the genuine suicide notes in Shneidman and Farberow's sample have anger in them directed toward a significant other, while none of the simulated suicide notes do so (Lester, 1989c). All this tells us is that nonsuicidal individuals do not realize that a small proportion of suicidal individuals are angry.

Instead, Lester suggested that simulated suicide notes are a good way to study the opinions and myths that people in the general population have about suicides and suicidal behavior. For example, in his sample of simulated notes, Lester found that women more often addressed their simulated suicide note to someone than did the men, apologized and asked forgiveness, stated that they were unhappy, and said that others would be better off if they were dead. Thus, men and woman appear to have different conceptions about the motives for suicide.

What might make a better comparison group for genuine suicide notes? Ideally, we need letters and notes written by these suicides at an earlier time when they were not suicidal. Then we could see how their psychological state had changed from the nonsuicidal period to the suicidal period. Unfortunately, most individuals do not have collections of letters and notes written over the course of their lifetime. Furthermore, regrettable though it is, researchers are sometimes lazy. It is easy to collect a sample of simulated suicide notes. For example, Shneidman and Farberow went to labor unions and fraternal groups and asked groups of men to write simulated suicide notes. I have asked students in my courses on suicide to write such notes. A captive group of people can write simulated suicide notes in ten minutes. To collect earlier letters from deceased suicides requires tracing the significant others of each suicide, visiting them, explaining the purpose of the research, seeing if they will cooperate by searching out earlier letters, if any exist, and providing counseling if they need it. Despite, Lester's objection to this research, this chapter will review the research

33 For each suicide note, an individual matched for age and occupational level was asked to write a suicide note. The sample was restricted to Caucasian, Protestant, native-born males aged 25 to 60. 
published on genuine versus simulated suicide notes and present the results of an LIWC analysis.

\subsection{Guessing which Notes are Genuine}

Can the genuine suicide note be distinguished from a simulated note? Osgood and Walker (1959) looked at the matched pairs of genuine and simulated notes published by Shneidman and Farberow. Osgood and Walker had graduate students with no prior experience of suicide notes predict which of each pair was the genuine note. The students performed at exactly the chance level, identifying a mean of 16.5 notes of the 33 pairs correctly. Osgood and Walker themselves carried out the task after reading and comparing a different sample of genuine suicide notes with a sample of ordinary letters. They were correct on 28.5 of the pairs. Therefore, experience with suicide notes increased the accuracy of assignment.

Lester (1993) obtained two sets of genuine and simulated suicide notes (one set from Shneidman and Farberow [1957b] and another set from Seiden and Tauber [1970]) and found that naive judges showed no parallel-forms reliability. He found that expert suicidologists could guess the genuine notes better than chance, while naive judges could not. Lester (1991a) had naive judges guess the genuine note in Shneidman and Farberow's sample of genuine and simulated note pairs, and the odd-even correlation was moderate, suggesting that the judges were reasonably consistent.

Lester (1991b) found the accuracy of naive judges of Shneidman and Farberow's sample of notes was associated with their extraversion scores, but not with their neuroticism or psychoticism scores or with their Keirsey-Bates temperament scores. Lester (1995a) found that Machiavellian scores did not predict the success of naive judges, while Lester (1994c) found that judges more sympathetic to suicide were more accurate in picking out the genuine notes, both studies again using Shneidman and Farberow's sample of notes.

Leenaars and Lester (1991) found that the genuine note in 19 of 33 pairs of genuine and simulated suicide notes was obvious to the student judges, while in 14 of the pairs it was not. Asking the judges for their reasons for judging a note to be genuine, it appeared that the students looked for traumatic events and idiosyncratic views to judge a suicide note to be genuine.

\subsection{Differences between Genuine and Simulated Suicide Notes}

Shneidman and Farberow (1957a) compared their genuine and simulated notes for the quality of the thought units in them using a coding system devised by Dollard and Mowrer (1947). The genuine notes had significantly more statements overall than the simulated notes, but did not differ in the proportion of discomfort statements 
(discomfort statements divided by the sum of relief plus discomfort statements). ${ }^{34}$ They noted informally that the type of discomfort statements differed in the genuine and simulated notes. The genuine notes had deeper feelings of hatred, vengeance and self-blame. However, this difference was not tested in a quantitative way by the authors. Shneidman and Farberow commented that the excess of neutral statements in the genuine notes possibly reflected a tendency on the part of the suicidal person to confuse the self as experienced by the self with the self as experienced by others, but I do not follow their reasoning here.

Spiegel and Neuringer (1963) argued that an individual does not necessarily complete suicide when the urge to die becomes stronger than the urge to live. What is necessary is that the dread that rises as the individual approaches a suicidal action needs to be reduced in some way. Spiegel and Neuringer argued that the defenses that are used to reduce dread might include self-deception over the imminent suicidal action, a tendency to avoid mention of suicide, and a tendency to concentrate on topics other than suicide. Psychotic processes, which would serve to protect the individual from realizing the implications of the suicidal action, might be reflected in the disorganization of the note.

These defenses were operationally measured by the following five attributes: relative explicitness of expressed suicidal intention, dramatic quality of the note, disorganization of thought processes, presence of the word "suicide" or suicide synonyms, and presence of instructions to the reader with respect to the disposal of property or the assumption of responsibilities. The results confirmed four of the five hypotheses: the genuine notes had less explicitness, fewer mentions of suicide, a greater number of instructions, and greater disorganization. There was no difference in dramatic quality.

Osgood and Walker (1959) argued that the suicidal individual has a heightened drive level, and they hypothesized that this would lead to greater stereotypy and greater disorganization in suicide notes than in ordinary letters. Since drive states are characterized by distinctive cues which have a directive function, they hypothesized that suicide notes should be characterized by increased frequency of those grammatical and lexical choices associated with motives leading to self-destruction. Finally, if several motives are operating, suicide notes should show greater evidence of conflict. To test these hypotheses, they compared a sample of suicide notes and a sample of ordinary letters written by nonsuicidal people matched for sex and age.

The suicide notes were more stereotyped than the letters: they had a smaller proportion of different words used, they had more repetition of phrases, there were fewer adjectival and adverbial qualifiers (as compared to the number of nouns and verbs), and they had more definitive terms (like always and never). The suicide notes of men were more redundant than the letters, but the difference for women was not

34 The statistical analysis presented in Shneidman and Farberow's paper appears to be incorrect. 
significant. There were no significant differences in the average number of syllables per word..$^{35}$

There were no differences between the suicide notes and the letters in disorganization-there were no differences in syntactical, grammar, spelling or punctuation errors. For women, there were no differences in the sentence length but, for men, the suicides notes had longer sentences, contrary to the prediction of Osgood and Walker who thought that, under stress, sentence length would decrease.

There was evidence of a directive state-the suicide notes had a higher proportion of discomfort statements, more evaluative common-meaning terms (like unfair and sweetheart), fewer positive evaluative assertions (a measure that is correlated with the proportion of discomfort statements), and more mands (utterances such as imperatives, which express a need of the speaker and require some reaction from the reader for their satisfaction). There was no difference in the distribution of past, present, and future references, whereas Osgood and Walker had predicted more emphasis on the past in suicide, notes. There was evidence for a state of conflict-the suicide notes had verbs with more qualifications (for example, "used to be good" rather than "was good"), more ambivalent constructions (such as but, should, maybe, and except), and more ambivalent evaluative assertions.

Osgood and Walker then applied their findings to Shneidman and Farberow's 33 pairs of genuine and simulated suicide notes. Only 13 pairs of notes had sufficient length for a meaningful analysis. For stereotypy, only the measure of adjectiveadverb/noun-verb ratio significantly differentiated the genuine from the simulated notes. (The redundancy measure was not used since the notes were too brief.) The mand measure of the directive state differentiated the two groups and, although the ambivalent construction measure of conflict differentiated the groups, the direction was opposite to that in the first part of the study. Disorganization measures were not investigated since they had not been found to be of use in the first part of the study. Eliminating those measures which failed to differentiate suicide notes from letters (structural disturbances, average length of sentences, and time orientation), those measures susceptible to faking (the proportion of discomfort statements, evaluative terms, and positive evaluative assertions), and the redundancy measure (since the notes were too brief for a meaningful application), Osgood and Walker applied the remaining nine measures to predict which of the notes in the 13 pairs were the genuine ones. The predictions were correct for 10 pairs, a significant difference.

To investigate further the stereotypy of the genuine notes, Osgood and Walker catalogued the words used by more than five of the writers of each group of notes. The genuine writers used more words in common than did the nonsuicidal writers. A content analysis showed that the genuine suicide notes had more terms of endearment

35 Osgood and Walker had argued that shorter words tend to be more frequent than longer words (Zipf, 1949) and so should indicated stereotypy. 
and references to mother, whereas the simulated notes contained more abstractions and references to insurance. Genuine notes had more verbs of simple action (go, tell) whereas simulated notes had more verbs referring to mental states (know, think). The genuine notes had more references to positive states (such as love and hope).

Osgood and Walker noted that possibly other models could have predicted their results. However, they felt that an explanation based on differences in educational and intelligence levels of the two groups of writers was unlikely to be valid since there were no differences in disorganization between the suicide notes and letters.

Tuckman and Ziegler (1966) examined the 33 pairs of genuine and simulated notes for differences in social maturity. They measured social maturity using the ontogenetic sequence observed by Piaget (1926) in the speech of children, in which the child moves away from the use of self terms (I, me) toward self-other terms (we, us) and finally to other terms (you, them). They predicted that the genuine notes should have a greater emphasis on self-reference than on other-reference. To test this, they counted the number of different categories of pronouns in the notes. The notes did not differ in the total number of different categories of pronouns or in the proportions. Tuckman and Ziegler concluded that the simulated notes may have been written in a state of anxiety and that this may have led to as low a level of social maturity as shown by the suicidal individuals.

Another study of content was carried out by Gottschalk and Gleser (1960) who had no hypotheses. They coded the words used in the 33 pairs of genuine and simulated notes into grammatical categories and psychological categories. The genuine notes had fewer prepositions, fewer conjunctions and more substantives than the simulated notes. There were no differences in the proportions of adjectives, adverbs, interjections, verbs, or total number of words. With regard to psychological categories, there were fewer words denoting reflective or cognitive process in the genuine notes. There were no differences in the proportion of words denoting feeling or motivation, perceptual processes, activity or movement, relationships in time or space, measures of quantity, or negation. Finally, there were no differences with regard to references to the self, animals or flowers, or to the person addressed. The genuine notes did have more references to others and to objects.

One complexity introduced by the authors in this study was that they analyzed the results in two ways: treating the notes as matched pairs and as independent groups. The results reported above were for matched-pairs analyses. The results of the analysis for independent groups differed. The only common significant differences were for substantives, references to others, and references to objects.

Gottschalk and Gleser then obtained a new sample of genuine suicide notes. For the signs that they had had identified in the first part of the study, no differences were found in the new sample for men as compared to women or for married people as compared to single people. When the new sample was compared to the simulated notes of the first part of the study, the results of the first part were replicated. Gottschalk and Gleser felt that the differences that they reported reflected the nature of 
the genuine suicide note as a communication to another, but they did not explain their reasoning here.

Ogilvie, et al. (1965) compared the same 33 pairs of genuine and simulated notes using a computer program to carry out a content analysis. The genuine notes had more references to "things" such as roles and objects. The simulated notes had more references to emotional states and actions. For the sentences containing a reference to the female role, it was found that the genuine note writes gave fewer instructions to females, gave more information about females, and made more references to being acted upon by a female than did the simulated note writers. ${ }^{36}$ Looking at the instructions given to females, the genuine note writers gave more specific instructions and fewer vague instructions.

Looking at actions, Ogilvie, et al. found that the genuine writers used the concept of "think" in the context of knowing or decision making ("I think that if I went to the doctor, I would. . . ), whereas the simulated note writers used the concept more in attempting to solve a problem ("I am thinking of all the problems we have shared"). Ogilvie, et al. noted the similarity of their results to those of Gottschalk and Gleser discussed above. Since the two sets of workers were using the same data and similar analyses, this is not surprising. Using three items (reference to concrete things, places and persons, use of the word love in the text, and total number of references to processes of thought and decision), the authors were able to classify 30 of the 33 pairs of notes correctly.

Henken (1976) used a computer program to compare the genuine suicide notes, simulated suicide notes and notes written by people about to die. The genuine notes were more concrete, constricted and concerned with interpersonal relationships (especially those with the opposite sex). Edelman and Renshaw (1982) also compared the genuine and simulated notes by computer analysis and found the genuine notes to be longer with fewer positive modifiers. The genuine notes had more negatives (e.g., "not”), authority (e.g., proper nouns), audience (e.g., "you”) and generalized others (e.g., third-person pronouns), modified nouns, modified verbs, cognizance of objects (concrete objects), modification of objects and actions, negative modification, negative modification of known people and positive modification of unknown people, and static action, and fewer references to future time.

Lester (1971b) found no difference between Shneidman and Farberow's genuine and simulated suicide notes in the need for affiliation expressed in them. Lester (1973) found no differences in the tense of the verbs in the notes, although the genuine notes did have more verbs in the conditional tense and more imperatives. Lester (1989c) found that the genuine notes more often had content reflecting the desire to kill (anger) but did not differ in the desires to be killed (depression and guilt) and to die (escape).

36 Remember, all of the note writers were male. 
Lester and Leenaars (1988) found that the genuine notes contained more direct accusations and wills/testaments and fewer conventional "first-form" notes (i.e., notes that focus on conventional explanations and pleas for forgiveness). McLister and Leenaars (1988; Leenaars, et al., 1989) found more signs of unconscious forces at play in the genuine notes than in the simulated notes (as well as more mention of spouses and fathers). Black (1993) found that the genuine notes were longer and, after controls for length, had more instructions, information, religious ideas and dates. The simulated notes had more depression, justification, life seen as overwhelming as a reason for suicide, and less mention of the after-life.

\subsection{Testing Alfred Adler's Theory of Suicide}

Darbonne (1967) compared a different sample of suicide notes written by a group of white American men who had completed suicide with simulated notes written by men matched for race, age, and occupation and simluated notes by white American men who were currently threatening suicide. The hypotheses tested by Darbonne derived from Adler's views on suicide (Ansbacher, 1961).

Adler maintained that the suicidal individual was characterized by a pampered life style and dependency. The notes of completed suicides as compared to the simulated notes more often mentioned a need to have things done for them, a heightened concern with whether or not they had received emotional support from others, and the importance of parental figures to them. There were no significant differences in the difficulties experienced in adapting to loss.

There was less support for the notion that the completed suicides would be characterized by inferiority feelings and self-centered goals. There were no differences between the two sets of notes in direct expressions of low self-esteem or self-praise, feelings of being at the mercy of external forces, or in the number of self-referents. However, there were more references by the completed suicides to other people than there were by the nonsuicidal persons, and there were also more mentions of the absence or breaking of social ties. Darbonne felt that these last two comparisons were relevant to feelings of inferiority and self-centered goals, but they seem to me to be more relevant to the dependency of the person.

Adler hypothesized that suicides would be very active, and Darbonne found that the completed suicides used more verbs referring to physical motor behavior than did the nonsuicidal persons, and their notes contained more verbs and adjectives. However, the notes did not differ in length (a measure of verbal activity), and there was no difference by the method of suicide used or chosen by the subject. (The methods of suicide had been previously rated by psychologists for the degree of activity involved.)

There was some support for the notion that the notes from completed suicides would show more veiled aggression than the simluated notes. The notes of the 
completed suicides had more veiled hostility, more frequent mentions of concern regarding the blame of others for their suicide, and more concern with informing others and addressing the note to others as compared to the simulated notes. However, the two sets of notes did not differ in expressions of direct anger, mentions of suffering, and feelings that others had been hurt by the past behavior of the note writer.

Overall, the results supported Darbonne's predictions based upon Adlerian ideas about suicide, with the exception of the hypothesized differences in inferiority feelings. Each idea tested by Darbonne received some support, and none of the tests produced significant results in a direction opposite to that predicted. The eleven variables that had proved successful in differentiating the notes of the completed suicides from those of the nonsuicidal persons were used to compare the notes of those threatening suicide with those of the two other groups. With regard to veiled aggression and a pampered life style and dependency, those threatening suicide resembled the completed suicides in inferiority feelings and self-centered goals but resembled the nonsuicidal persons in activity. Those threatening suicide were divided into a high risk and a low risk group. The two groups did not differ on any of the eleven variables significantly. The resemblance of those threatening suicide with the completed suicides perhaps reflects the degree to which those threatening suicide possess high suicidal potential. However, the comparison of the high and low risk group among those threatening suicide failed to reveal differences, and so interpretation of the results from those threatening suicide is not easy.

\subsection{Testing Other Theories of Suicide}

Leenaars formulated several theories of suicide into specific statements and examined the presence of these statements in Shneidman and Farberow's 33 pairs of genuine and simulated suicide notes. Leenaars (1988a) found that genuine notes contained more of the themes based on the theories of Sigmund Freud, Edwin Shneidman, and Gregory Zilboorg, and to a lesser extent Henry Murray and Harry Sullivan, but not from the theories of Alfred Adler, Ludwig Binswanger, Carl Jung, or George Kelly. Balance and Leenaars (1986) found that the genuine and simulated notes had different frequencies for statements derived from Aaron Beck's theory of suicide. The genuine notes had more magnification, minimization and overgeneralization, while the simulated notes had more hopelessness, suffering and desire for escape. Lester and Leenaars (1987) compared the genuine notes of each pair which naïve subjects could easily identify correctly with those which were hard to identify. The protocol sentences used by Leenaars in his research failed to differentiate these two sets of genuine notes. 


\subsection{Handwriting}

Frederick (1968) investigated whether suicide notes could be distinguished using handwriting. He had a sample of genuine notes typed and had handwritten copies made from the typed copies by individuals matched with the suicidal writers for sex and age. The paper used for the note was matched with the original note for size, color, type of paper, and amount of wear. Frederick used three sets of judges. Graphologists identified the genuine notes at a level better than chance, while detectives and secretaries both performed at chance level. There was no apparent effect on the accuracy of the judgments from the type of paper used for the note or from the sex and age of the writers.

\subsection{The LIWC Analysis}

The 33 pairs of genuine and simulated suicide notes from the book written by Shneidman and Farberow (1957b) were run through the LIWC. All were from Caucasian, Protestant, native-born men ages 25-59 years. Shneidman and Farberow matched the writers of these genuine and simulated for age and occupation, and so the scores can be treated as paired data.

Paired t-tests identified 10 significant differences out of 76 (see Table 11.1). The genuine notes were longer and had fewer words per sentence, fewer unique words, more pronouns, more second person pronouns such as "you," fewer prepositions, more numbers, fewer words related to causation such as "because" and "hence," more words concerned with social processes such as "talk" and "friend," and more references to people. It appears, therefore, that the genuine notes were more concerned with other people and less focused on explaining why the individual is choosing to die by suicide. Interestingly, there were no differences in content categories, including school or work, leisure activities, religion, death and dying, body states and symptoms, or sex and sexuality. ${ }^{37}$

These results did not confirm the differences reported by Osgood and Walker (1959) reviewed above in positive emotions or optimism, but the results did confirm that the genuine notes had fewer references to cognitive processes and causation. Unlike the results reported by Tuckman and Ziegler (1966) who found no differences in pronouns, the present analysis found more references to "you" in the genuine notes. However, Gottschalk and Gleser's (1960) results concerning pronouns did match the present results.

37 Treating the data as two independent samples and using point-biserial correlation coefficients, the same 10 significant differences were identified as those found in the paired-sample analysis. 
Tab. 11.1: Differences between simulated and genuine notes (mean scores shown)

\begin{tabular}{lll}
\hline & Genuine notes & Simulated notes \\
\hline Word count & 108.88 & $65.67^{\star}$ \\
Words per sentence & 10.90 & $14.35^{\star}$ \\
Unique words & 71.24 & $80.12^{\star}$ \\
Pronouns & 20.62 & $16.68^{\star}$ \\
Total second person & 5.43 & $3.38^{\star}$ \\
Prepositions & 9.16 & $11.65^{\star}$ \\
Numbers & 1.08 & $0.28^{\star}$ \\
Causation & 0.50 & $1.53^{\star}$ \\
Social processes & 12.37 & $8.71^{\star \star}$ \\
Other references to people & 7.81 & $5.27^{\star}$ \\
\hline * two-tailed $p<.05,{ }^{* \star}$ two-tailed $\mathrm{p}<.01$ & \\
\hline
\end{tabular}

\subsection{A Second Sample of Simulated Suicide Notes}

Seiden and Tauber (1970) also collected a sample of genuine and simulated suicide notes, and these notes were also analyzed by the LIWC program (see Table 11.2). There were 17 significant differences. The genuine suicide notes had shorter sentences, fewer dictionary words, less use of "we," fewer articles and prepositions, more use of numbers, more affect (emotion) and especially positive emotions, less use of cognitive mechanisms, including causation and discrepancies, more tentative words, more references to seeing, more references to family, and more words concerned with "down" and "exclusion."

Which differences were similar in this set of genuine and simulated suicide notes and those from Shneidman and Farberow? In both sets, the genuine notes had shorter sentences, fewer prepositions and more numbers, and less concern with causation. It appears, then, that simulated note writers try to explain the reasons for their suicide more than the genuine notes writers and write longer sentences in order to do this. The greater use of numbers in the genuine suicide notes may reflect the greater amount of instructions and directions (such as whom to contact and to whom various objects and money is to be given). The numbers were most likely telephone numbers and street addresses. 


\subsection{Pseudocides}

Seiden and Tauber (1970) also collected a sample of notes left by people who had possibly pretended to kill themselves from the Golden Gate Bridge. These cases had no eye-witness to the jump and no body was found. From 134 possible cases, 34 appeared to be "suspicious," and 24 of these were established as "definite." Ten of these were

Tab. 11.2: A comparison of genuine, simulated and hoax suicide notes (point biserial correlation shown)

\begin{tabular}{|c|c|c|c|}
\hline & $\begin{array}{l}\text { Genuine v. } \\
\text { Simulated } \\
\text { notes }\end{array}$ & $\begin{array}{l}\text { Hoax v. } \\
\text { simulated } \\
\text { notes }\end{array}$ & $\begin{array}{l}\text { Hoax v. } \\
\text { genuine } \\
\text { notes }\end{array}$ \\
\hline Word count & -0.22 & $0.28^{\#}$ & $0.37^{\star}$ \\
\hline Words per sentence & $-0.53^{\star \star}$ & $-0.52^{\star \star}$ & 0.04 \\
\hline Unique words & $0.29^{\#}$ & -0.10 & $-0.32^{\star}$ \\
\hline Words in the dictionary & $-0.50^{\star \star}$ & $-0.33^{\star}$ & $0.29^{\#}$ \\
\hline Words longer than 6 letters & -0.04 & $-0.37^{\star}$ & -0.25 \\
\hline First person plural & $-0.32^{\star}$ & $-0.30^{\#}$ & 0.20 \\
\hline Total third person & -0.15 & 0.17 & $0.38^{\star}$ \\
\hline Articles & $-0.48^{\star *}$ & -0.05 & $0.39^{\star \star}$ \\
\hline Prepositions & $-0.54^{\star \star}$ & $-0.34^{\star}$ & 0.22 \\
\hline Numbers & $0.33^{\star}$ & $0.31^{*}$ & -0.22 \\
\hline Affect & $0.33^{\star}$ & 0.23 & -0.18 \\
\hline Positive emotions & $0.37^{\star}$ & 0.14 & $-0.27^{\#}$ \\
\hline Positive feelings & $0.29^{\#}$ & 0.15 & -0.14 \\
\hline Cognitive processes & $-0.56^{\star \star}$ & $-0.34^{\star}$ & $0.34^{\star}$ \\
\hline Causation & $-0.35^{\star}$ & $-0.29^{\#}$ & 0.13 \\
\hline Insight & $-0.30^{\#}$ & $-0.34^{\star}$ & 0.05 \\
\hline Discrepancies & $-0.46^{\star \star}$ & -0.01 & $0.43^{\star \star}$ \\
\hline Tentative & $-0.36^{\star}$ & -0.20 & 0.20 \\
\hline Seeing & $-0.31^{\star}$ & -0.09 & 0.25 \\
\hline Family & $0.31^{\star}$ & 0.01 & $-0.31^{\star}$ \\
\hline Future tense verbs & $-0.27^{\#}$ & 0.01 & 0.26 \\
\hline Down & $-0.31^{\star}$ & -0.08 & $0.38^{\star \star}$ \\
\hline Exclusive & $-0.48^{\star \star}$ & -0.08 & $0.38^{\star}$ \\
\hline School & 0.16 & $0.36^{*}$ & 0.26 \\
\hline Religion & $0.28^{\#}$ & 0.18 & -0.21 \\
\hline Death & -0.11 & $-0.37^{\star}$ & -0.13 \\
\hline Eating & - & $0.28^{\#}$ & $0.28^{\#}$ \\
\hline Sexual & $0.30^{\#}$ & 0.19 & -0.12 \\
\hline
\end{tabular}


later arrested for crimes other than being a missing person. These pseudocides showed little depression prior to their action or social withdrawal. They tended to be impulsive individuals who acted-out non-violently.

Seiden and Tauber reported that the pseudocide notes from 21 individuals were longer than the genuine and simulated notes, although with a much greater variability in length. The pseudocide notes gave more realistic reasons for their suicide (such as mentioning financial and legal problems more), contained more emotion, and were more explicit about their suicide as compared to the genuine notes. Lester, et al. (1990) found that anger (Menninger's “to kill” motive) was present more often in the pseudocide notes than in the genuine or simulated notes, while self-blame (Menninger's "to be killed" motive) was present more often in the genuine suicide notes and the desire to escape less often present.

The 21 pseudocide notes were compared, using the LIWC program with the 21 genuine and the 21 simulated notes from the same study, and the results are shown in Table 11.2. The pseudocide and simulated notes differed significantly on ten variables, while the pseudocide and genuine notes differed significantly on nine variables. Thus, the pseudocide notes did not resemble the genuine suicide notes either more or less than they resembled the simulated suicide notes. They appear to constitute a third type of note, different in many respects from both genuine and simulated suicide notes.

This makes sense since the writers of pseudocide notes are, presumably, not suicidal, and so their notes should differ from genuine suicide notes. On the other hand, they are probably in the midst of a major crisis and feeling desperate since they are faking their own suicide and starting a new life with a different identity. Thus, their notes should differ from simulated suicide notes which are typically written by people who are neither suicidal nor in a major crisis. 